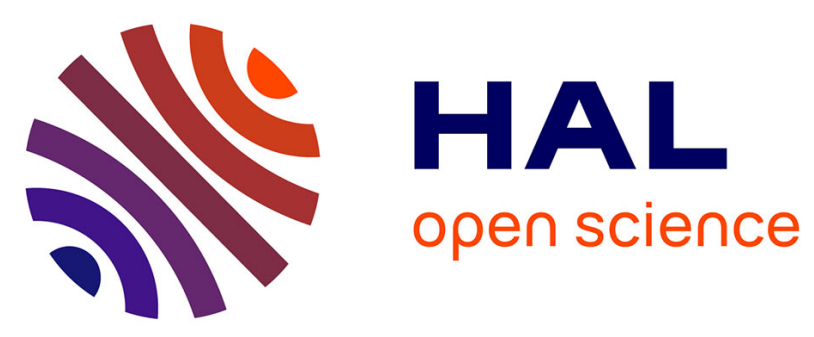

\title{
Generalising Conservativity
}

Richard Zuber

\section{- To cite this version:}

Richard Zuber. Generalising Conservativity. WoLLIC 2010, 2010, France. pp.247-258. halshs00751201

\section{HAL Id: halshs-00751201 https://shs.hal.science/halshs-00751201}

Submitted on 15 Nov 2012

HAL is a multi-disciplinary open access archive for the deposit and dissemination of scientific research documents, whether they are published or not. The documents may come from teaching and research institutions in France or abroad, or from public or private research centers.
L'archive ouverte pluridisciplinaire HAL, est destinée au dépôt et à la diffusion de documents scientifiques de niveau recherche, publiés ou non, émanant des établissements d'enseignement et de recherche français ou étrangers, des laboratoires publics ou privés. 


\title{
Generalising Conservativity
}

\author{
R.Zuber \\ Rayé des cadres du CNRS \\ Richard.Zuber@linguist.jussieu.fr
}

\begin{abstract}
A constraint on functions from sets and relations to sets is studied. This constraint is a generalisation of the constraint of conservativity known from the study of generalised quantifiers in natural languages. It is suggested that this generalised constraint constitutes a semantic universal.
\end{abstract}

\section{Introduction}

Progress in the study of the logical properties of natural languages (NLs) is closely related to the study of various constraints that must be satisfied by functions interpreting functional expressions in NLs. We know that such expressions do not denote arbitrarily and thus that functions interpreting them obey various specific logic constraints. The most prominent results obtained in this context are obtained in generalised quantifier theory and concern functions corresponding to various types of quantifiers. The constraint on quantifiers which has been extensively studied from theoretical and empirical points of view is the constraint of conservativity, which concerns the denotations of various determiners found in NLs. Conservativity is generally considered as a language universal. Even though some non-conservative determiners are known, it appears that they are rare and not arbitrary since they are systematically related to conservative determiners (cf. Zuber 2004).

In this paper I generalise the notion of conservativity so that it can apply not only to quantifiers but also to some functions having sets as results. Furthermore, I suggest that the constraint of conservativity thus generalised also constitutes a language universal.

Let me make first some notational and definitional preliminaries which will allow us to define the properties and functions which we are going to discuss. We will be interested in sets and relations over a given universe $E$. If $R$ is a $k+n$-ary relation and $a_{1}, \ldots, a_{k} \in E$ then $a_{1} \ldots a_{k} R$ is the $n$-ary relation defined as follows:

$$
a_{1} \ldots a_{k} R=\left\{\left\langle a_{k+1}, \ldots, a_{n}\right\rangle \in E^{n}:\left\langle a_{1} \ldots a_{k+n}\right\rangle \in R\right\}
$$

The functions in which we will be interested are functions which have as inputs (arguments) sets and relations. If the output of such a function is a truthvalue then this function is a (generalised) quantifier. A type $\langle 1\rangle$ quantifier is a 
function from sets to truth-values. It is a denotation of a noun phrase (used in subject position in a sentence). Type $\langle 1,1\rangle$ quantifiers are functions from binary relations between sets to truth values or just binary relations between sets. Type $\langle 1,2\rangle$ quantifiers are binary relations between sets and binary relations (that is relations whose domains are sets and co-domains - binary relations between sets). Finally type $\langle 1,1,2\rangle$ (or type $\left\langle 1^{2}, 2\right\rangle$ ) quantifiers are ternary relations whose first two "arguments" are sets and the third argument is a binary relation between sets.

We will discuss basically functions which are not quantifiers though they can be seen as related to quantifiers. They are not quantifiers because their codomains are not truth-values but sets (or relations in a more general case). The type of function which takes $n$ sets and one $k$-ary relation and gives a $i$-ary relation (for $i<k$ ) as result will be noted $\left\langle 1^{n}, k: i\right\rangle$, for $n \geq 1$. The type of function taking a n-ary relation and giving as result an i-ary relation (for $i<k$ ) will be noted $\langle n: i\rangle$. These functions will be called arity reducers.

A special class of arity reducers is obtained from quantifiers. Since type $\langle m\rangle$ quantifiers are functions from $m$-ary relations to truth-values, in order to consider them more generally as arity reducers we need to define their extensions, that is to extend theirs domains so that they can apply to relations of any arity (higher than $m$ ). This can be done in two steps in the following way (cf. Keenan and Westerståhl 1997):

D1: Let $R_{n}$ be the set of n-ary relations (over E). Then $F$ is a reducer of arity by k, noted $F \in\left[\bigcup_{n} R_{n+k} \rightarrow \bigcup R_{n}\right]$ is in $A R(-k)$, if $\forall R \in R_{n+k}, F(R) \in R_{n}$, $(n \geq 0)$.

Every type $\langle k\rangle$ quantifier extends to a function in $A R(-k)$ in the following way:

D2: A type $\langle k\rangle$ quantifier $Q$ extends to a function in $A R(-k)$, also noted $Q$, by letting, for $R \in R_{n+k}, Q(R)=\left\{\left\langle a_{1}, \ldots, a_{n}\right\rangle \in E^{n}: Q\left(a_{1} \ldots a_{n} R\right)=1\right\}$

Thus by D2 any type $\langle k\rangle$ quantifier can be considered as a type $\langle n+k: n\rangle$ function, that is a function which applies to relations of arity $n+k$ and which gives as a result a relation of arity $n$.

Of course there are functions which are arity reducers but which are not extensions of quantifiers. Extended type $\langle 1\rangle$ quantifiers are characterised by the following property (Keenan and Westerståhl 1997):

Proposition 1: Let $F \in A R(-1) . F$ is extended type $\langle 1\rangle$ quantifier iff $\forall n, m \geq 0$, $\forall R \in R_{n+1} \forall S \in R_{m+1} \forall a_{1}, \ldots, a_{n}, b_{1}, \ldots, b_{m} \in E$, we have

$$
a_{1} \ldots a_{n} R=b_{1} \ldots b_{m} S \Rightarrow\left(\left\langle a_{1}, \ldots, a_{n}\right\rangle \in F(R) \Leftrightarrow\left\langle b_{1}, \ldots, b_{m}\right\rangle \in F(S)\right)
$$


From now on we will be interested in the particular case when $k=2, n \leq 2$ and $i=1$ and thus we will study basically functions of type $\langle 2: 1\rangle$, of type $\langle 1,2: 1\rangle$ and to a lesser extend functions of type $\left\langle 1^{2}, 2: 1\right\rangle$.

Let me illustrate some of the above notions. We are interested in the interpretation of simple sentences of the form $N P_{1} T V P N P_{2}$. In such sentences the noun phrase $N P_{1}$ is interpreted by a type $\langle 1\rangle$ quantifier, which is a set of sets, and the transitive verb phrase $T V P$ is interpreted by a binary relation. Concerning $\mathrm{NP}_{2}$ there are two possibilities: it can be interpreted either by a function which is an extension of a type $\langle 1\rangle$ quantifier or by a function (from binary relations to sets) which is not an extension of a type $\langle 1\rangle$ quantifier. When such a function is an extension of a quantifier we will call it accusative extension (since it occurs in the direct object position of a sentence) and note it $Q_{a c c}$. It is an instance of D2 and is specified in D3:

D3: If $Q$ is a type $\langle 1\rangle$ quantifier, then $Q_{a c c}(R)=\{a: Q(a R)=1\}$.

Obviously accusative case extensions satisfy the invariant condition given in Proposition 1 and which for the case we consider $(\mathrm{k}=2)$ will be called the accusative extension condition (AEC):

AEC: A function $F$ from binary relations to sets satisfies the AEC iff for $R$ and $S$ binary relations, and $a, b \in E$, if $a R=b S$ then $a \in F(R)$ iff $b \in F(S)$.

Thus type $\langle 1\rangle$ quantifiers become type $\langle 2: 1\rangle$ functions by accusative extension. It is also true, however, that any type $\langle 2: 1\rangle$ function which satisfies the AEC condition uniquely determines a type $\langle 1\rangle$ quantifier. Indeed the following proposition holds:

Proposition 2: If a type $\langle 2: 1\rangle$ function $F$ satisfies the AEC and the type $\langle 1\rangle$ quantifier $Q$ is defined as $Q(X)=1$ iff $a \in F(\{a\} \times X)$ then $Q_{a c c}(R)=F(R)$

Proof:

Type $\langle 1\rangle$ quantifiers and type $\langle 2: 1\rangle$ functions satisfying AEC form complete atomic Boolean algebras. For any set $A \subseteq E$, the function $F_{A}$ such $F_{A}(X)=1$ iff $X=A$ is an atom of the algebra of type $\langle 1\rangle$ quantifiers. Similarly, the function $F_{A}$ such that $F_{A}(R)=\{x: x R=A\}$ is an atom of the second algebra. These two algebras are isomorphic (the mapping given in D3 establishes the isomorphism). Given that $F_{A}(\{a\} \times A)=A$ if $a \in A$ and $F_{A}(\{a\} \times A)=\emptyset$ if $a \notin A$, we get the needed result.

The following example illustrates Proposition 2. Consider a type $\langle 2: 1\rangle$ function $F_{A}$ defined as follows: $F_{A}(R)=\left\{x:|x R \cap A|>\left|x R^{\prime} \cap A\right|\right\}$ (for a fixed $A \subseteq E$ and $E$ finite). Since $a R=b S$ iff $a R^{\prime}=b S^{\prime}$ the function $F_{A}$ satisfies AEC. It is easy to see that $F_{A}=(\operatorname{MOST}(A))_{\text {acc }}$ (where $\operatorname{MOST}(A)(Y)=1$ iff $\left.|A \cap Y|>\left|A \cap Y^{\prime}\right|\right)$. 
The following two simple conditions can be used to decide whether functions from binary relations to sets satisfy or not the AEC condition:

Fact 1: A type $\langle 2: 1\rangle$ function $F$ does not satisfy the AEC if there exists a set $B$ and $a, b \in B$ such that $a \in F(E \times B) \wedge b \notin F(E \times B)$

Fact 2: If a type $\langle 2: 1\rangle$ function $F$ satisfies AEC then for any $A \subseteq E$ one has $F(E \times A)=\emptyset$ or $F(E \times A)=E$.

In the next section we consider two classes of functions which need not satisfy the AEC condition.

\section{Some arity reducers}

Not every function from binary relations to sets used in NLs semantics satisfies AEC. The best known example is the function $S E L F$ defined as $S E L F(R)=$ $\{x:\langle x, x\rangle \in R\} ; S E L F$ can be used to interpret the reflexive pronoun him/herself.

Among functions not satisfying AEC one can distinguish two sub-classes (cf. Keenan and Westerståhl 1997), according to whether they satisfy one of the two conditions which are strictly weaker than AEC. Because such functions are most frequently found in the semantics because they are necessary for the interpretation of various expressions in NLs, I briefly introduce them.

The function $S E L F$ satisfies the the so-called anaphor condition AC:

AC: A function $F$ from binary relations to sets satisfies the anaphor condition iff for $R$ and $S$ binary relations, and $a \in E$, if $a R=a S$ then $a \in F(R)$ iff $a \in F(S)$.

Obviously, functions which satisfy AEC also satisfy AC. Functions which satisfy AC but do not satisfy AEC will be called anaphoric functions.

One can check (Keenan 2007) that $S E L F$ is an anaphoric function of type $\langle 2: 1\rangle$ as is the function NOBODY-EXCEPT-SELF needed to interpret sentence $(1)$ :

(1) Leo hates everybody except himself.

The AC applies to functions of type $\langle 2: 1\rangle$. We need a similar condition for type $\langle 1,2: 1\rangle$ functions (which in NLs are basically denotations of anaphoric determiners):

ACD1: A function $F$ of type $\langle 1,2: 1\rangle$ satisfies anaphor condition (ACD1) iff for any $a \in E, X \subseteq E$ and $R, S$ binary relations, if $a(E \times X \cap R)=a(E \times X \cap S)$ then $a \in F(X, R)$ iff $a \in F(X, S)$.

To illustrate ACD1 consider the following example: 
(2) Leo trusts no philosopher except himself.

In this example the function $N O-E X C E P T-S E L F$ of type $\langle 1,2: 1\rangle$ defined in (3) is involved:

(3) $F(X, R)=N O(X)-E X C E P T-S E L F(R)=\{y: y R \cap X=\{y\}\}$

One can check that this function satisfies ACD1.

The following property gives a justification of the condition ACD1:

Fact 3: If the function $F$ of type $\langle 1,2: 1\rangle$ satisfies ACD1 then the function $G^{A}$ of type $\langle 2: 1\rangle$ defined as $G^{A}(R)=F(A, R)$ satisfies AC.

What fact 3 informally says is that functions satisfying ACD1 are those from which we get functions satisfying $\mathrm{AC}$ when fixing their set argument.

We can use the same method of fixing "nominal" arguments to define anaphoric functions of type $\langle 1,2: 1\rangle$. Thus we have:

D4: A function $F$ of type $\langle 1,2: 1\rangle$ is anaphoric iff it satisfies the condition ACD1 and the function $G^{A}$ of type $\langle 2: 1\rangle$ defined as $G^{A}(R)=F(A, R)$ is anaphoric for any non-trivial $A$.

Using fact 1 one can show that the function defined in (3) is anaphoric.

In the above definitions, anaphoricity of type $\langle 1,2: 1\rangle$ functions is reduced to anaphoricity of type $\langle 2: 1\rangle$ functions. The condition of non-triviality for $A$ and $B$ is necessary because obviously for values of $A$ or $B$ making $F$ constant such that we get functions which also satisfy AEC.

Let us consider now the second weakening of the AEC, the so-called argument invariance (Keenan and Westerståhl 1997):

D5: A type $\langle 2: 1\rangle$ function $F$ is argument invariant iff whenever $a R=b R$ then $a \in F(R)$ iff $b \in F(R)$.

The above definition obviously generalises to type $\langle 1,2: 1\rangle$ functions:

D6: A type $\langle 1,2: 1\rangle$ function $F$ is argument invariant iff whenever $a(E \times X \cap R)=$ $b(E \times X \cap R)$ then $a \in R(X, R)$ iff $b \in F(X, R)$

As an example consider the function involved in the interpretation of the numerical superlative the greatest number of as it occurs in (4a) with the intended meaning given in (4b). This superlative is interpreted by the function given in (5):

(4a) Leo knows the greatest number of languages.

(4b) Leo knows more languages than anybody else. 
(5) $N S U P(X, R)=\{x: \forall y(y \neq x \rightarrow|x R \cap X|>|y R \cap X|\}$

It is easy to check that $N S U P$ is argument invariant and that it does not satisfy the AEC1 condition.

\section{Conservativity}

Conservativity is a property of some classes of quantifiers. It has been basically studied in connection with type $\langle 1,1\rangle$ quantifiers but a non-trivial notion of conservativity applies to many classes of quantifiers which take at least two arguments. In particular quantifiers denoted by unary and n-ary determiners can be said to be conservative. Moreover Westeståhl 2004 shows how to define conservativity for type $\left\langle 1^{m}, k\right\rangle$ quantifiers. Conservativity of some other classes of quantifiers is defined in Keenan and Westerståhl (1997).

Conservativity of quantifiers denoted by unary or n-ary determiners (that is determiners taking $n$ common nouns to form a noun phrase) can be easily defined, as we will see. We have seen that anaphoric functions (that we consider here) are systematically related to quantifiers (of type $\langle 1,1\rangle$ ). Simularly, "comparative" functions which are argument invariant (and which interpret comparative constructions such as the one a in (5)), seem to be related to quantifiers. So it is quite natural to ask whether and in what sense anaphoric functions are conservative.

Let us recall first the notion of conservativity for type $\langle 1,1\rangle$ quantifiers. A now well-known definition is given in D5:

D5: $F \in C O N S$ iff for any property $X, Y$ one has $F(X)(Y)=F(X)(X \cap Y)$

Given D5 it is easy to show that the type $\langle 1,1\rangle$ quantifier $F$ defined as $F(X)(Y)=$ 1 iff $X=Y$, is not conservative.

Conservativity of type $\langle 1,1\rangle$ quantifiers can additionally be formulated in two different ways:

Fact 5 (cf. Keenan and Faltz 1986) $: F$ is conservative or $F \in C O N S$ iff for any property $X, Y$ and $Z$ if $X \cap Y=X \cap Z$ then $F(X)(Y)=F(X)(Z)$

Fact 6 (Zuber 2005): $F \in C O N S$ iff for any property $X, Y$ one has $F(X)(Y)=$ $F(X)\left(X^{\prime} \cup Y\right)$

It is also possible to define conservativity for the whole class of type $\langle 1,1,2\rangle$ quantifiers. In this case we have the following definition, an instance of the general definition proposed in Westerståhl 2004:

D6: A type $\langle 1,1,2\rangle$ quantifier $F$ is conservative iff for any sets $A, B$ and any binary relation $R$ one has $F(A, B, R)=F(A, B, A \times B \cap R)$ 
As in the case of "simple" type $\langle 1,1\rangle$ quantifiers it is possible to give an equivalent defining condition for conservativity of type $\langle 1,1,2\rangle$ quantifiers to hold. Thus we have:

Proposition 3: A type $\langle 1,1,2\rangle$ quantifier is conservative iff $F\left(A, B, R_{1}\right)=F\left(A, B, R_{2}\right)$ whenever $A \times B \cap R_{1}=A \times B \cap R_{2}$

Clearly none of the above definitions of conservativity applies directly to an anphoric function. However, proposition 3 and fact 5 give us a hint as to what form the definition of conservativity of type $\langle 1,2: 1\rangle$ functions should take. Here is the definition:

D7: Let $F$ be a type $\langle 1,2: 1\rangle$ function. Then $F$ is conservative iff for all $X \subseteq E$ and $R_{1}, R_{2}$ binary relations, if $E \times X \cap R_{1}=E \times X \cap R_{2}$ then $F\left(X, R_{1}\right)=F\left(X, R_{2}\right)$.

By analogy with fact 1 and definition D1 conservativity of type $\langle 1,2: 1\rangle$ functions can be defined equivalently as the following proposition shows:

Proposition 4: A function $F$ of type $\langle 1,2: 1\rangle$ is conservative iff $F(X, R)=$ $F(X, E \times X \cap R)$

It is easy to check that the anaphoric type $\langle 1,2: 1\rangle$ function $E V E R Y$ $E X C E P T-S E L F$ defined above, is conservative.

The following property gives additional plausibility to the above definitions of generalised conservativity:

Proposition 5: Let $D$ be a type $\langle 1,1\rangle$ quantifier and $F$ a type $\langle 1,2: 1\rangle$ function defined as: $F(X, R)=D(X)_{a c c}(R)$. Then $F$ is conservative iff $D$ is conservative.

Proof:

Suppose a contrario that $F$ is conservative and $D$ is not. Thus for some $X, Y \in E$, $D(X)(Y) \neq D(X)(X \cap Y)$. Let $R=E \times Y$. Then:

$F(X, R)=D(X)_{a c c}(R)=\{a: D(X)(a R)=1\}=\{a: D(X)(a(E \times Y))=1\}$

$F(X, E \times X \cap R)=D(X)_{a c c}(E \times(X \cap Y))=\{a: D(X)(a(E \times(X \cap Y)))=1\}$ Since $D(X)(a(E \times Y))=D(X)(Y)$ and $D(X)(a(E \times(X \cap Y)))=D(X)(X \cap Y)$, this means that $F(X, R) \neq F(X, E \times X \cap R)$, which is impossible given that $F$ is conservative.

Suppose now that $D$ is conservative. Then:

$F(X, R)=D(X)_{a c c}(R)=\{a: D(X)(a R)=1\}=$

$=\{a: D(X)(X \cap a R)=1\}$, since $D$ is conservative

$=\{a: D(X)(a(E \times X \cap R)=1\}$, since $X=a(E \times X)$ and $a(R \cap S)=a R \cap a S)$

$=D(X)_{a c c}(E \times X \cap R)=F(X, E \times X \cap R)$ 
Thus the generalised conservativity of functions induced by type $\langle 1,1\rangle$ quantifiers, when they are used in the accusative extension of a type $\langle 1\rangle$ quantifier, is strictly related to the "classical" conservativity of the inducing quantifier.

Given the example of non-conservative type $\langle 1,1\rangle$ quantifier given above it follows from Proposition 5 that the type $\langle 1,2: 1\rangle$ function $F$ defined as $F(X, R)=\{y: X=y R\}$ is not conservative.

Let us recall now some properties of denotations of binary determiners, that is quantifiers of type $\langle\langle 1,1\rangle 1\rangle$. We have the following definition of conservativity (Keenan and Moss 1985, Zuber 2005):

D8: A type $\langle\langle 1,1\rangle 1\rangle$ quantifier is conservative iff for any $X_{1}, X_{2}, Y_{1}, Y_{2} \subseteq E$, if $X_{1} \cap Y_{1}=X_{1} \cap Y_{2}$ and $X_{2} \cap Y_{1}=X_{2} \cap Y_{2}$ then $F\left(X_{1}, X_{2}\right)\left(Y_{1}\right)=F\left(X_{1}, X_{2}\right)\left(Y_{2}\right)$.

The following proposition shows the equivalent way to define conservativity for type $\langle\langle 1,1\rangle 1\rangle$ quantifiers:

Proposition 6: A type $\langle\langle 1,1\rangle 1\rangle$ quantifier is conservative iff for any $X_{1}, X_{2}, Y \subseteq E$ one has $F\left(X_{1}, X_{2}\right)(Y)=F\left(X_{1}, X_{2}\right)\left(Y \cap\left(X_{1} \cup X_{2}\right)\right)$.

Definition D8 and proposition 6 can be used as basis for generalising conservativity to type $\left\langle 1^{2}, 2: 1\right\rangle$ functions:

D9: A type $\left\langle 1^{2}, 2: 1\right\rangle$ function $F$ is conservative iff for any $X_{1}, X_{2} \subseteq E$ and any binary relations $R_{1}$ and $R_{2}$, if $E \times X_{1} \cap R_{1}=E \times X_{1} \cap R_{2}$ and $E \times X_{2} \cap R_{1}=$ $E \times X_{2} \cap R_{2}$ then $F\left(X_{1}, X_{2}, R_{1}\right)=F\left(X_{1}, X_{2}, R_{2}\right)$.

The corresponding equivalent property is indicated in the following proposition:

Proposition 7: A type $\left\langle 1^{2}, 2: 1\right\rangle$ function $F$ is conservative iff for any $X_{1}, X_{2} \subseteq E$ and binary relation $R$ one has $F\left(X_{1}, X_{2}, R\right)=F\left(X_{1}, X_{2},\left(E \times\left(X_{1} \cup X_{2}\right)\right) \cap R\right)$.

Let us see some examples. As we know the type $\langle\langle 1,1\rangle 1\rangle$ quantifier $\operatorname{MORE}\left(X_{1^{-}}\right.$ THAN $\left(X_{2}\right)$ is denoted by the binary determiner more...than.... This determiner may form a noun phrase with two common names. This noun phrase can occur in object position as in (6):

(6) Leo knows more logicians than philosophers.

One can consider that in this case the type $\langle\langle 1,1\rangle 1\rangle$ quantifier $\operatorname{MORE}\left(X_{1}\right)$ THAN $\left(X_{2}\right)$ gives rise to a type $\left\langle 1^{2}, 2: 1\right\rangle$ function $F$ defined in (7):

(7) $F\left(X_{1}, X_{2}, R\right)=\left(\operatorname{MORE}\left(X_{1}\right)-T H A N\left(X_{2}\right)\right)_{a c c}(R)=\left\{y:\left|y R \cap X_{1}\right|>\mid y R \cap\right.$ $\left.X_{2} \mid\right\}$ 
This function is conservative. This is not surprising since $\operatorname{MORE}\left(X_{1}\right)-T H A N\left(X_{2}\right)$ is conservative and we have:

Proposition 8: Let $D$ be a type $\langle\langle 1,1\rangle 1\rangle$ quantifier. Then the type $\left\langle 1^{2}, 2: 1\right\rangle$ function $F\left(X_{1}, X_{2}, R\right)=\left(D\left(X_{1}, X_{2}\right)\right)_{a c c}(R)$ is conservative iff $D$ is conservative.

Proof of Proposition 8 is similar to that of Proposition 5.

One can consider that Proposition 8 "justifies" D9.

There are obviously type $\left\langle 1^{2}, 2: 1\right\rangle$ functions which are not obtained by the accusative extension of type $\langle\langle 1,1\rangle 1\rangle$ quantifiers. Consider the example in (8) which involves the type $\left\langle 1^{2}, 2: 1\right\rangle$ function given in (9):

(8) Leo knows more languages than Adam (knows) theorems.

(9) $F_{a}\left(X_{1}, X_{2}, R\right)=\left\{y:\left|y R \cap X_{1}\right|>a R \cap X_{2} \mid\right\}$

The function $F_{a}$ is conservative (and comparative).

\section{Other constraints}

Generalised conservativity as introduced in the previous section in D7 and D9 concerns type $\langle 1,2: 1\rangle$ and type $\left\langle 1^{2}, 2: 1\right\rangle$ functions in general and not only anaphoric or comparative functions. Moreover generalised consevativity is independent of anaphor conditions ACD1 and ACD2 for anaphoric functions. It is also independent of argument invariance. What is interesting is the fact that some anaphoric functions satisfy also other constraints, some of which are stronger than generalised conservativity.

Observe that type $\langle 1,2: 1\rangle$ anaphoric functions discussed above, for instance the function given in (3), are used to interpret various constructions in which the reflexive pronoun him/her-self occurs. such functions satisfy the constraint given in (8):

(8) $F(A, R) \subseteq A$.

Interestingly, the anaphoric condition ACD1, (generalised) conservativity and the condition given in (8) entail a specific version of conservativity, anaphoric conservativity (or a-conservativity), specific to self-type anaphoric determiners. It is defined in D10:

D10: A type $\langle 1,2: 1\rangle$ function $F$ is a-conservative iff $F(X, R)=F(X, X \times X \cap R)$.

Thus we have the following proposition:

Proposition 9: A type $\langle 1,2: 1\rangle$ anaphoric and conservative function $F$ such that $F(X, R) \subseteq X$ is a-conservative. 
Proof: Suppose a contrario that for some $X \subseteq E, F(X, R) \neq F(X, X \times X \cap R)$ and thus that (by conservativity) $F(X, E \times X \cap R) \neq F(X, X \times X \cap R)$. This means that for some $a \in X, a \in F(X, E \times X \cap R)$ and $a \notin F(X, X \times X \cap R)$ (or $a \notin F(X, E \times X \cap R)$ and $a \in F(X, X \times X \cap R))$. This is, however, impossible given that $F$ is anaphoric and the fact that in this case $a(E \times X \cap R)=a(X \times X \cap R)$.

It follows from the observations made above that self-type anaphoric functions described above are a-conservative. There are also anaphoric functions which are not a-conservative (Zuber 2010).

It is well-known that various natural language quantifiers can satisfy stronger constraints than conservativity (Keenan 1993). In particular they can be intersective or co-intersective. These sub-classes of quantifiers are theoretically important for various reasons. For instance Keenan 1993 shows that conservative type $\langle 1,1\rangle$ quantifiers are Booleanly generated by intersective and co-intersective quantifiers. They are also of empirical interest since they lead to various linguistically relevant generalisations (cf. Peters and Westerståhl 2006, Kuroda 2008).

The question thus arises whether one can generalise the notion of intersectivity or co-intersectivity to some functions which are not quantifiers. In what follows I show briefly how it can be done.

Recall that a type $\langle 1,1\rangle$ quantifier $D$ is intersective (resp. co-intersective) iff $D\left(X_{1}, Y_{1}\right)=D\left(X_{2}, Y_{2}\right)$ whenever $X_{1} \cap Y_{1}=X_{2} \cap Y_{2}$ (resp. $X_{1} \cap Y_{1}^{\prime}=X_{2} \cap Y_{2}^{\prime}$. This leads to the following definitions of intersective or co-intersective anaphoric functions:

D11: A type $\langle 1,2: 1\rangle$ function is intersective (resp. co-intersective) iff $F\left(X_{1}, R_{1}\right)=$ $F\left(X_{2}, R_{2}\right)$ whenever $E \times X_{1} \cap R_{1}=E \times X_{2} \cap R_{2}$ (resp. $\left.E \times X_{1} \cap R_{1}^{\prime}=E \times X_{2} \cap R_{2}^{\prime}\right)$.

The following proposition, similar to Proposition 5, can be considered as justifying the above definition:

Proposition 10: Let $D$ be a type $\langle 1,1\rangle$ quantifier and $F$ a type $\langle 1,2: 1\rangle$ function defined as: $F(X, R)=D(X)_{a c c}(R)$. Then $F$ is intersective (resp. co-intersective) iff $D$ is intersective (resp. co-intersective).

It is easy to see that the function $N O(X)-B U T-S E L F(R)$ as defined in (3) above is intersective. Similarly, the function $F_{a}$, for $a \in E$, defined in (9) and which is necessary to interpret (10), is an intersective function:

(9) $F_{a}(X, R)=\{y:|y R \cap X| \geq|a R \cap X|\}$

(10) Leo knows at least as many languages as Adam.

Concerning co-intersective functions it is easy to show that the function $E V E R Y(X)-B U T-S E L F(R)$ defined in (11) is co-intersective: 
(11) $\operatorname{EVERY(X)-BUT-SELF(R)}=\left\{x: X \cap x R^{\prime}=\{x\}\right\}$

It is also possible to generalise other sub-properties of conservativity. Consider so-called cardinal quantifiers. A type $\langle 1,1\rangle$ quantifier $F$ is cardinal iff $F\left(X_{1}\right)\left(Y_{1}\right)=F\left(X_{2}\right)\left(Y_{2}\right)$ whenever $\left|X_{1} \cap Y_{1}\right|=\left|X_{2} \cap Y_{2}\right|$. For instance numerals denote cardinal quantifiers.

In order to generalise the property of cardinality (of quantifiers) to type $\langle 1,2: 1\rangle$ functions observe first the following equivalence:

(12) $E \times X_{1} \cap R_{1}=E \times X_{2} \cap R_{2}$ iff $\forall y\left(X_{1} \cap y R_{1}=X_{2} \cap y R_{2}\right)$

This means that the condition in D11 can be replaced by the right hand side expression in (12). This leads to the following definition:

D12: A type $\langle 1,2: 1\rangle$ function is cardinal iff $F\left(X_{1}, R_{1}\right)=F\left(X_{2}, R_{2}\right)$ whenever $\forall y\left(\left|X_{1} \cap y R_{1}\right|=\left|X_{2} \cap y R_{2}\right|\right)$

For cardinal functions we have the following proposition, similar to proposition 5 and proposition 10 :

Proposition 11: Let $D$ be a type $\langle 1,1\rangle$ quantifier and $F$ a type $\langle 1,2: 1\rangle$ function defined as: $F(X, R)=D(X)_{a c c}(R)$. Then $F$ is cardinal iff $D$ is cardinal.

The function given in (9) is a cardinal function which is not obtained by the accusative case extension. Similarly, the comparative function $F_{a}$ given in (13) needed to interpret (14) is a cardinal function (not obtained by the accusative case extension of a cardinal quantifier):

(13) $F_{a}(X, R)=\{y:|y R \cap X|>|a R \cap X|\}$

(14) Leo proved more theorems than Adam.

Obviously, cardinal functions are conservative. This means that the function $F(X, R)=\{y: X=y R\}$ is not cardinal.

\section{Conclusion}

After having recalled the various properties of anaphoric and comparative functions which represent the biggest class of type $\langle 1,2: 1\rangle$ functions found in NLs, I have proposed a generalisation of the notion of conservativity classically used in the context of quantifiers. Moreover, some notions stronger than conservativity, that is intersectivity and cardinality (of quantifiers) are also generalised to specific functions. Conservativity is a very natural property. In simple cases it has empirical and theoretical justification (Peters and Westerståhl 2006). In the domain of determiners, that is expressions denoting, roughly speaking, quantifiers, 
it is considered as a language universal. This means that one has enough empirical data to consider that all determiners, defined syntactically, in all natural languages denote only conservative quantifiers.

Even if in any serious (composional) semantics the (complex) expressions discussed here will automatically get the generalised conservativity (if at least the (simple) quantifiers that are used as building blocks are conservative), it is very tempting to make a similar universalistic claim about the generalised conservativity of specific functions from sets and relations to sets studied in this article. In other words, one would like to suggest, roughly, that NLs expressions denoting type $\langle 1,2: 1\rangle$ or type $\left\langle 1^{2}, 2: 1\right\rangle$ functions always denote conservative functions.

Any serious defence of such a claim should be preceded by additional research along the following two lines. First, obviously, more empirical research should be done. The notion of generalised conservativity proposed here applies to all type $\langle 1,2: 1\rangle$ functions, not only anaphoric ones or comparative ones. It has been supposed here that the functions which are not obtained by a case extension and known to be needed in the semantics of NLs are either anaphoric (cf. Zuber 2010) or comparative. This supposition should be empirically substantiated. Second, a syntactic description of expressions denoting conservative functions should be provided. The underlying idea in this article is that such functions are denotations of anaphors or of comparatives and superlatives. Thus the precise syntactic status of such constructions should be provided. This syntactic part of the enterprise is considered as being outside the scope of this article.

\section{References}

1. Keenan, E. L. (1993) Natural Language, Sortal Reducibility and Generalised Quantifiers, Journal of Symbolic Logic 58:1, pp. 314-325.

2. Keenan, E. L. (2007) On the denotations of anaphors. Research on Language and Computation 5-1: 5-17

3. Keenan, E. L. and Faltz, L. M. (1985) Boolean Semantics for Natural Language, D. Reidel Publishing Company, Dordrecht.

4. Keenan, E. L. and Moss, L. (1985) Generalized quantifiers and the expressive power of natural language, in J. van Benthem and A. ter Meulen (eds.) Generalized Quantifiers, Foris, Dordrecht, pp.73-124

5. Keenan, E. L. and Westerståhl, D. (1997) Generalized Quantifiers in Linguistics and Logic, in van Benthem, J. and ter Meulen, A. (eds.) Handbook of logic and language, Elsevier, Amsterdam, pp. 837-893

6. Peters, S. and Westerståhl, D. (2006) Quantifiers in Language and Logic, Clarendon Press, Oxford

7. Kuroda, S-Y. (2008) Head Internal Relative Clauses, Quantifier Float, the Definiteness Effect and the Mathematics of Determiners, San Diego Linguistics Papers 3, pp. $126-183$

8. Westerståhl, D. (1994) Iterated Quantifiers, in Kanazawa, M. and Pinon, Ch. (eds.) Dynamics, Polarity and Quantification, CSLI, Stanford University, pp. 173-209

9. Zuber, R. (2004) A class of non-conservative determiners in Polish, Linguisticae Investigationes, XXVII : 1, pp. 147-165 
10. Zuber, R. (2005) More Algebras for Determiners, in P. Blache and E. Stabler (eds.) Logical Aspects of Computational Linguistics 5, LNAI, vol. 3492, Springer-Verlag, pp. $363-378$

11. Zuber, R. (2010) Semantics of Slavic anaphoric possessive determiners, Proceedings of SALT 19 